\title{
OPPORTUNITIES TO INTERCALIBRATE RADIOMETRIC SENSORS FROM INTERNATIONAL SPACE STATION*
}

\author{
C. M. Roithmayrł C. Lukashin \\ K. J. Thome, \\ D. F. Young, and B. A. Wielicki**
}

\begin{abstract}
Highly accurate measurements of Earth's thermal infrared and reflected solar radiation are required for detecting and predicting long-term climate change. We consider the concept of using the International Space Station to test instruments and techniques that would eventually be used on a dedicated mission such as the Climate Absolute Radiance and Refractivity Observatory. In particular, a quantitative investigation is performed to determine whether it is possible to use measurements obtained with a highly accurate reflected solar radiation spectrometer to calibrate similar, less accurate instruments in other low Earth orbits. Estimates of numbers of samples useful for intercalibration are made with the aid of year-long simulations of orbital motion. We conclude that the International Space Station orbit is ideally suited for the purpose of intercalibration.
\end{abstract}

\section{INTRODUCTION}

It is essential that we understand the nature of the changes taking place in Earth's climate, correctly attribute the changes to natural variability or anthropogenic effects, make reliable long-term forecasts for the magnitude of those changes, and act accordingly. We must have high confidence in our predictions of climate because our response may entail enormous societal and economic costs.

Earth's thermal infrared and reflected solar radiation are among the quantities that must be measured accurately on a global scale over long time spans in order to gain the necessary understanding and ability to predict climate change. Current methods of measuring these two variables from spacecraft are not sufficiently accurate; an order of magnitude improvement is required. Two proposed complementary missions address the need for greater accuracy by employing instruments with onboard calibration that permits traceability to international metrological standards. The first of these, Climate Absolute Radiance and Refractivity Observatory (CLARREO) (Reference 1), is one of

\footnotetext{
${ }^{*}$ This material is declared a work of the U.S. Government and is not subject to copyright protection in the United States.

${ }^{\dagger}$ Senior Aerospace Engineer, Vehicle Analysis Branch, NASA Langley Research Center, Hampton, Virginia 23681.

${ }^{\ddagger}$ Research Physical Scientist, Climate Science Branch, NASA Langley Research Center, Hampton, Virginia 23681.

${ }^{\S}$ Research Aerospace Engineer, Space Mission Analysis Branch, NASA Langley Research Center, Hampton, Virginia 23681.

`CLARREO Deputy Project Scientist, Biospheric Sciences Branch, NASA Goddard Space Flight Center, Greenbelt, Maryland 20770

" CLARREO Project Scientist, Climate Science Branch, NASA Langley Research Center, Hampton, Virginia 23681.

** Senior Scientist for Radiation Sciences, Climate Science Branch, NASA Langley Research Center, Hampton, Virginia 23681.
} 
the four top-priority missions recommended in the 2007 Decadal Survey report (Reference 2) by the U.S. National Research Council. The second, Traceable Radiometry Underpinning Terrestrialand Helio- Studies (TRUTHS) (Reference 3), is proposed by the United Kingdom National Physical Laboratory. The highly accurate measurements obtained from such missions are important not only for their intrinsic value as climate benchmarks, but also for their usefulness in calibrating less accurate radiometric instruments aboard other spacecraft. Through a process known as reference intercalibration, the accuracy of other instruments can be improved significantly, thereby enhancing the performance of an entire climate observation system. Intercalibration requires detailed data matching; that is, measurements from two spacecraft must be taken along similar lines of sight, and within a few minutes of each other. Similar techniques are currently used for intercalibration of orbiting satellite sensors as part of the Global Space-based Inter-Calibration System (GSICS), an international effort to improve the consistency of satellite intercalibration. The current system, however, lacks an orbiting high-accuracy SI-traceable reference radiometer comparable to CLARREO. GSICS has indicated measurements from such an instrument form a critical element in anchoring satellite observations, especially for climate applications (Reference 4).

Despite a well-established need, recognition of the important advantages, and extensive preparatory research conducted thus far, there are currently no firm plans for dedicated satellite missions such as CLARREO and TRUTHS. Considerable thought is, however, being given to placing CLARREO instruments on alternative orbiting platforms in order to test equipment and techniques, and make it possible to obtain the benefits of highly accurate measurements before dedicated spacecraft can be put into service. In this regard the International Space Station (ISS) appears to be an attractive site, for several reasons.

First, there is at present significant interest in conducting studies of global climate change from the ISS, as evidenced by the Announcement of Opportunity (Reference 5) issued by the European Space Agency in 2011, and a large number of proposals received in response to a preceding call for ideas in 2009. The NASA Science Mission Directorate provided a similar avenue in 2011 with the Earth Venture - 2 Announcement of Opportunity (Reference 6). Second, in connection with measurements of thermal infrared and reflected solar radiation specifically, an advantageous relationship exists between the ISS orbit and the sun-synchronous orbits in which radiometric sensors are often placed. The orbit plane of ISS precesses westward with respect to an inertial frame of reference, completing a full revolution in about 72 days. The period of eastward precession for the orbit plane of a sun-synchronous spacecraft is, of course, one year. Consequently, alignment of the ascending nodes of the two orbits goes through a cycle whose period is 60 days; thus, measurements can be compared for viewing geometry conditions that recur every 60 days. Furthermore, the ISS orbital altitude is approximately $400 \mathrm{~km}$ less than that of key sun-synchronous satellites, resulting in relatively gradual changes in direction of the line of sight between spacecraft. Third, the long projected service life of ISS, together with the presence of a human crew and robotic equipment, make it possible to consider operational options that will not be available at other spacecraft. In particular, failed equipment can be serviced and repaired, the instrument can be replaced every few years on a regular basis, and the instrument can be returned to Earth for post-flight evaluations and possible refurbishment. All of the aforementioned considerations indicate ISS can be a useful stepping-stone on the way to dedicated missions such as CLARREO and TRUTHS.

As a precursor to a free-flying CLARREO mission, two top-of-atmosphere instruments could be placed on ISS: a thermal infrared upward radiation spectrometer, and a reflected solar radiation spectrometer. These instruments would serve the international community by intercalibrating other 
Earth observing instruments, including, but not limited to: Advanced Baseline Imager (ABI), Advanced Geostationary Radiation Imager (AGRI), Advanced Himawari Imager (AHI), Atmospheric Sounding Interferometer (ASI), Advanced Very High Resolution Radiometer (AVHRR), Clouds and the Earth's Radiant Energy System (CERES), Cross-track Infrared Sounder (CrIS), Enhanced Thematic Mapper + (ETM+), Flexible Combined Imager (FCI), Geostationary Interferometric Infrared Sounder (GIIRS), Global Ozone Monitoring Experiment - 2 (GOME-2), High-resolution Infra Red Sounder (HIRS), Infrared Atmospheric Sounding Interferometer (IASI), Infra Red Atmospheric Sounder (IRAS), Infra Red Sounder (IRS), Medium Resolution Spectral Imager - 2 (MERSI-2), Moderate-resolution Imaging Spectro-radiometer (MODIS), Ocean and Land Colour Imager (OLCI), Spinning Enhanced Visible Infra-Red Imager (SEVIRI), Second-generation Global Imager (SGLI), Sea and Land Surface Temperature Radiometer (SLSTR), Visible/Infrared Imager Radiometer Suite (VIIRS), and Visible and Infra Red Radiometer (VIRR).

Several of the foregoing instruments are in Geosynchronous Earth Orbit (GEO). In some respects, it is more straightforward to intercalibrate these instruments than sensors in low Earth orbit. Three or four intercalibration opportunities arise each day, and a single opportunity can last more than 20 minutes. Intercalibration of GEO sensors will be considered in future work (Reference 7); however, the forthcoming discussion here is limited to intercalibration of instruments in low Earth orbit.

In this paper we restrict our attention to the reflected solar radiation spectrometer, and perform a quantitative examination of opportunities to intercalibrate other reflected solar radiation instruments placed aboard two particular sun-synchronous spacecraft. The first of these is the Joint Polar Satellite System (JPSS), which passes through its ascending node at 1:30 pm local time; the second spacecraft is MetOp, whose local time of ascending node is 9:30 pm. The JPSS instruments of interest are VIIRS and CERES, whereas MetOp carries the AVHRR. Each of these instruments scans in the crosstrack direction (perpendicular to the ground track of the host spacecraft), and our analysis is tailored to such behavior. Simulations of orbital motion over one-year intervals have been conducted to quantify geographic position, duration, and viewing angles of the measurements, as well as motion required of a two-degree-of-freedom instrument gimbal. Estimates are provided for monthly and seasonal numbers of ISS measurement samples that can be used to intercalibrate the aforementioned instruments.

The remainder of the paper consists of five sections. In the first of these we describe the CLARREO reflected solar radiation spectrometer in some detail, provide a definition of intercalibration, and determine the numbers of samples required for intercalibrating instruments on JPSS and MetOp. The second and third sections contain, respectively, a description of simulations of orbital motion, and a presentation of simulation results. Estimates of the numbers of samples that can be obtained from the ISS orbit are given in the fourth section, and conclusions are provided in the final section.

\section{CLARREO REFERENCE INTERCALIBRATION GOALS AND METHODOLOGY}

In order to investigate whether or not it is possible to perform intercalibration of reflected solar radiation instruments with measurements obtained from the ISS, we must first determine the numbers of samples (averaged groups of measurements) required to characterize parameters of interest for those instruments. Before doing so, we describe the CLARREO reflected solar radiation spectrometer requirements and features, and provide a technical definition of intercalibration. 


\section{CLARREO Reflected Solar Hyper-Spectral Imager}

Intercalibration methodology is intimately related to CLARREO reflected solar (RS) mission requirements and instrument features. The basis of the design of the RS sensor is the retrieval of an at-sensor reflectance over the spectral range from $320 \mathrm{~nm}$ to $2300 \mathrm{~nm}$, with a $0.5-\mathrm{km}$ groundprojected instantaneous field of view and a $100-\mathrm{km}$ swath width at nadir. Measurements of radiance while viewing the Earth's surface will be converted to a reflectance through ratios of solar-based measurements. The measurement signal will vary by factors of 2 to 10 because it is functionally dependent on solar zenith angle, wavelength, atmospheric gas absorption that changes with altitude and wavelength, and scene type that ranges from dark (clear-sky ocean) to bright (deep convective clouds). The RS instrument must be designed to account for these effects, and to include a calibration approach that allows accurate retrieval of the reflectance in the middle of the visible part of the spectrum, traceable to International System of Units (SI) standards at a level better than $0.3 \%(k=2)$ (References 2, 3, and 8). (We use $k$ instead of $\sigma$ to establish a rigorous tie between the climate science and metrology research communities. In the case of a Gaussian distribution, $k=1$ signifies the same confidence level as $1 \sigma$.) This accuracy is required to drive decadal climate change observational uncertainties to levels smaller than the internal natural variability of the climate system (References 1, 3, and 9). The driving instrument requirements for the RS sensor are summarized in Table 1, where the solar zenith angle, $\theta_{0}$, at a point on Earth's surface is defined as the angle between local zenith and the line of sight to the Sun.

Table 1. Driving requirements for CLARREO RS instrument.

\begin{tabular}{l|l}
\hline Parameter & Instrument Requirement \\
\hline Radiometric Accuracy & $0.3 \%(k=2)$ in broadband reflectance \\
Spectral Range & $320 \mathrm{~nm}$ to $2300 \mathrm{~nm}$ \\
Spectral Sampling & $4 \mathrm{~nm}$ \\
Instantaneous Field of View & $0.5 \mathrm{~km} \times 0.5 \mathrm{~km}$ \\
Nadir Swath Width & $100 \mathrm{~km}$ \\
Signal-to-Noise Ratio & $>33\left(\right.$ for mean 0.3 reflectance at $\left.\theta_{0}=75^{\circ}\right)$ \\
Sensitivity & Increases at longer wavelengths \\
\hline
\end{tabular}

The primary data product from the RS instrument is spectral reflectance. While the term reflectance has many meanings related to reflectance distribution functions, reflectance factors, and reflectivity, the current operational plan for the RS instrument is to determine the ratio of the output of the instrument while viewing an Earth scene to that of the instrument while viewing the Sun. Taking into account the geometric differences between a radiance measurement (while viewing the Earth scene) and an irradiance measurement (the solar measurement) permits the retrieval of a directional-hemispheric reflectance.

The need to measure the surfaces of the Sun and Earth means that the signals will vary by up to 5 orders of magnitude ( 1 to 50,000). Given the focus on spectral reflectance, the RS sensor will function like a band-ratioing radiometer. The instrument is based on an Offner imaging spectrometer design, which is capable of limiting spectral smile on the focal plane. The instrument will operate as a hyperspectral pushbroom imaging spectrometer; it relies on two separate focal planes based on silicon and MgCdTe detectors coupled with existing Indigo 9803 read-out integrated circuits having 
$640 \times 512$ pixels. Each spectrometer has its own separate entrance aperture, grating, and focal plane.

Both spectrometers are nearly identical in size, optical train, and mass. Each camera will face nadir and be equipped with a front-mounted, rotatable attenuator wheel and sunshield. A single detector plane thermal radiator cools both focal planes. In the two-box design, the blue spectrometer covers $320 \mathrm{~nm}-640 \mathrm{~nm}$ and the red spectrometer covers $600 \mathrm{~nm}-2300 \mathrm{~nm}$. Due to its relatively narrow spectral range, the blue spectrometer will use a conventional, single blaze diffraction grating. In contrast, the broad spectral range of the red/near-infrared spectrometer necessitates the use of a double blaze diffraction grating, coupled with an order-sorting filter. The overall mass of a single spectrometer is $20 \mathrm{~kg}$. The integrated instrument is mounted on a two-degree-of-freedom gimbal that allows it to match the line of sight of those sensors being intercalibrated, and therefore match the solar zenith angles associated with the measurements.

As mentioned earlier, such an approach requires that the sensor be able to reduce the incident solar energy to a level comparable to the Earth-viewing energy, by approximately a factor of 50,000. The attenuator approaches being evaluated include a single pinhole aperture, neutral density filters, a collection of pinhole apertures, or combinations of these three. The reason that three attenuator approaches are currently under study is that an additional goal of CLARREO calibration is to rely on multiple and independent calibration approaches. The attenuators require extremely careful evaluation during ground testing, and are also a source of uncertainty on orbit if the attenuators degrade in some fashion. Evaluation of the attenuators on orbit takes place through coordinated views of the Sun and the Moon. The brightness of the Moon is low enough to permit measurements without the attenuators in place, allowing coupled lunar and solar views to be used to determine if the attenuators are operating properly.

\section{CLARREO RS Reference Intercalibration Approach}

We define reference intercalibration as multi-dimensional minimization of the difference between calibrated sensor measurements, and CLARREO RS spectrometer high-accuracy SI-traceable measurements, performed for all available matched sampling. Intercalibrated instrument response parameters are relative to the CLARREO SI-traceable reference and derived over a time period (month, season, year). The intercalibration approach is based on retrieval of instrument offset and gain for every intercalibration data configuration, by comparison with CLARREO as a high-accuracy reference. It is essential that every intercalibration data configuration is provided with adequate sampling to reduce matching noise between CLARREO and intercalibrated sensor data, and to allow offset and gain retrieval with uncertainty below $0.3 \%(k=2)$ over a climate autocorrelation time period of 18 months (Reference 9).

\section{CLARREO RS Reference Intercalibration Objectives}

We prioritize CLARREO intercalibration tasks separately for CERES, and for the VIIRS and AVHRR imagers. High-priority intercalibration tasks are summarized in Table 2 for these instruments, with an indication of required intercalibration time scale and variable for data stratification. The required limit on the intercalibration error contribution is based on the assumption that all monthly and seasonal calibrations are independent over a climate autocorrelation time period of 18 months. $N$ denotes the number of intercalibration samples required to reduce the error to the stated value. 
Table 2. Summary of high-priority intercalibration tasks. Viewing zenith angle is denoted as $\theta$, degree of linear polarization as $P$. Relative error contribution from reference intercalibration in $\%(k=2)$ for corresponding time period, and required intercalibration sample number $N$.

\begin{tabular}{l|l|c|c|c|r}
\hline Sensor & Parameter & Time Scale & Variable & $\begin{array}{c}\text { Error }(\%) \\
(k=2)\end{array}$ & $N$ \\
\hline CERES & Offset & monthly & scan angle & 1.2 & $1.25 \times 10^{3}$ \\
& Gain & monthly & scan angle & 1.2 & $1.25 \times 10^{3}$ \\
& Degradation of optics & seasonally & scene type & 0.7 & $15 \times 10^{3}$ \\
& Non-linearity & annually & all data & 0.3 & $150 \times 10^{3}$ \\
\hline VIIRS, \& & Baseline offset & monthly & $P$, scan angle & 1.2 & $5 \times 10^{3}$ \\
AVHRR & Baseline gain & monthly & $P$, scan angle & 1.2 & $5 \times 10^{3}$ \\
& Sensitivity to polarization & seasonally & $P, \theta$ & 0.7 & $900 \times 10^{3}$ \\
& Non-linearity & annually & $P$, scan angle & 0.3 & $500 \times 10^{3}$ \\
\hline
\end{tabular}

The highest priorities for CERES intercalibration are gain, scan-angle-dependent electronic offset, and correction of spectral response function (Reference 10). CERES bolometer detectors are designed and verified to achieve less than $0.1 \%$ nonlinearity, and the spherical symmetry of the Cassegrain optics is designed to eliminate polarization sensitivity (Reference 11). The spectral response function is very broad ( $350 \mathrm{~nm}$ to $3500 \mathrm{~nm}$ wavelength) but optics contamination for all reflected solar sensors typically occurs below $500 \mathrm{~nm}$; therefore, spectral response must be verified for achieving climate accuracy.

For CLARREO intercalibration of VIIRS and AVHRR, the highest priorities are the baseline offset, gain, nonlinearity, and gain corrections due to sensitivity to polarization. MODIS sensitivity to polarization is reported to be $2 \%-4 \%$, depending on band and viewing geometry (Reference 12). Sensitivity to polarization is a response of the optical system, and it results in an additional contribution to effective gain, depending on scene type and viewing geometry. One important characterization of viewing geometry is the viewing zenith angle, $\theta$, defined at a point on Earth's surface to be the angle between local zenith and the line of sight to the orbiting instrument of interest. The strong angular dependence of Earth scene degree of polarization (Reference 13) makes it necessary to change the direction in which the CLARREO RS sensor is aimed, in order to intercalibrate imager sensitivity to polarization. This is accomplished by mounting the RS sensor on a two-degree-offreedom gimbal, as discussed presently. Change in the imager optics response function, such as a central wavelength shift of narrowband, is reported to be small for both MODIS instruments on Terra and Aqua platforms (References 14 and 15), and, therefore, its detection is not given a high priority.

\section{DESCRIPTION OF ORBITAL SIMULATIONS}

As discussed in Reference 16, analysis of opportunities for intercalibration over long periods is made computationally efficient by adopting several simplifying assumptions regarding orbital motions of two spacecraft about Earth, Earth's orbit about the Sun, and Earth's shape. In short, all orbits are treated as circular, and Earth is considered to have a spherical surface. Precession of a spacecraft's orbit plane with respect to an inertial reference frame is taken into account by employing a well-known expression for orbital average nodal regression rate as a function of inclination of the 


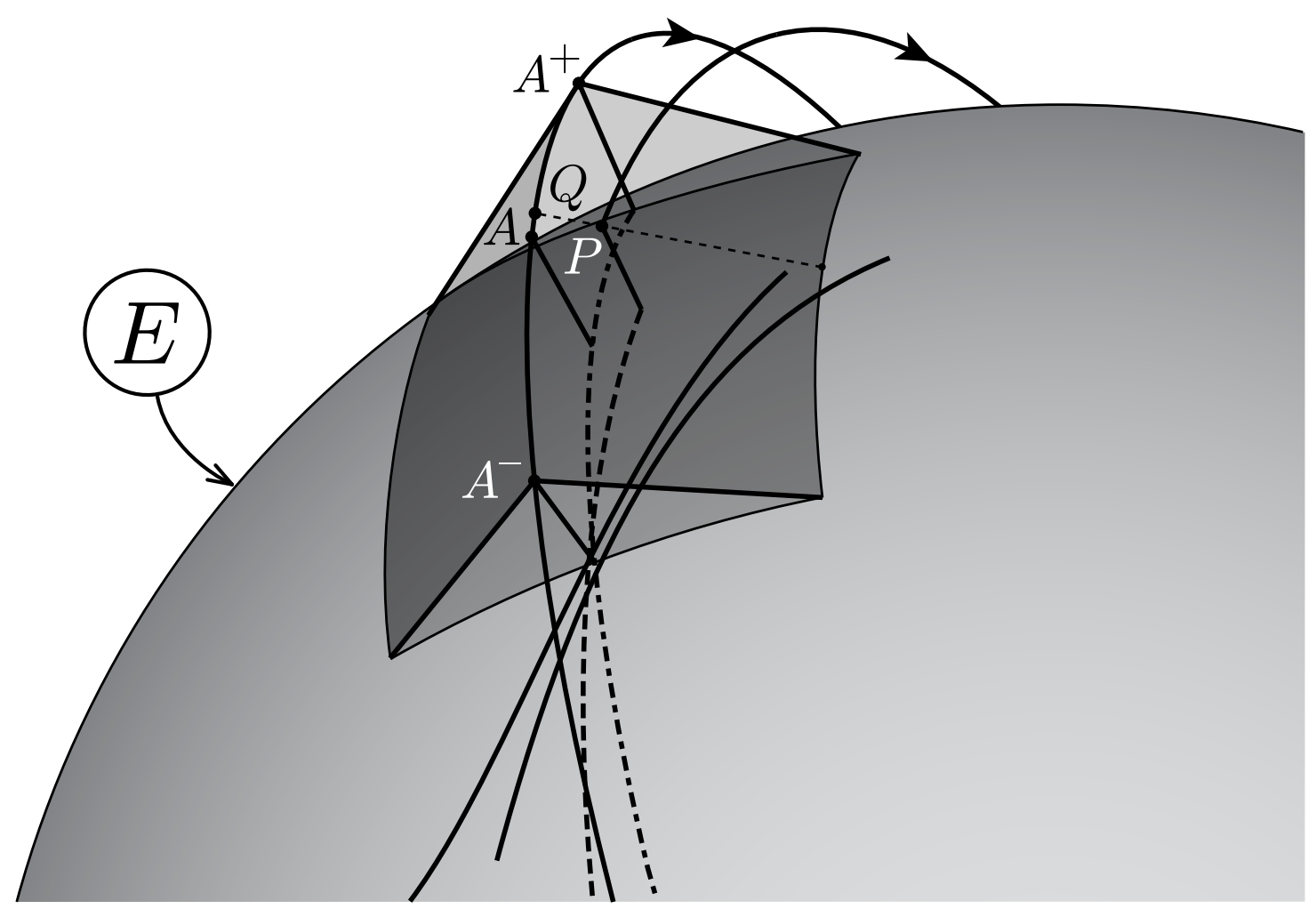

Figure 1. Intercalibration Tent

orbit plane, radius of the orbit, and the parameter $J_{2}$ that quantifies Earth's oblate mass distribution. For convenience, each simulation begins at the instant of autumnal equinox.

Because measurements of reflected solar radiation are of interest, solar illumination of Earth's surface must be considered when determining whether or not an opportunity for intercalibration exists. For the purposes of the analysis discussed here, a necessary condition for intercalibration is that the subsatellite points of both spacecraft must lie on the illuminated hemisphere, to include the terminator.

Provided the foregoing condition of illumination is satisfied, an intercalibration opportunity is regarded as the period of time during which ISS is inside a "tent" that is attached to the second spacecraft, say, JPSS, as illustrated in Figure 1. The field of view of the JPSS instrument has an angular displacement to either side of nadir; this scan angle, taken here to be $55^{\circ}$, determines the slope of the roof of the tent. Reflected solar radiation lies in the shortwave region of the spectrum and the scenes observed by the instrument change rapidly; consequently, a limit is imposed on the time elapsed between measurements obtained by the two spacecraft (Reference 17). Analysis is performed in this study with a temporal constraint of five minutes. In Figure 1, point $A$ denotes the actual position of JPSS. The temporal constraint is imposed by imagining a spacecraft $A^{+}$that is five minutes ahead of $A$ in its orbit, and another spacecraft $A^{-}$that is five minutes behind $A$. The arc that passes through $A^{+}, A$, and $A^{-}$forms the ridge of the tent's roof. When ISS, denoted by $P$, is inside the tent, an intercalibration opportunity exists. At any instant, one point $Q$ on the ridge of the tent is closest to $P$. The ISS sensor is aimed toward Earth, $E$, such that the boresight is parallel to the line joining $Q$ and $P$; thus, at the resulting target on the surface of $E$, the viewing zenith angle to $P$ (the 
angle $\theta$ between local zenith and the line of sight to $P$ ) is the same as the viewing zenith angle to $Q$. The boundaries of the ISS instrument swath are indicated with solid black curves. As measurement points in the instrument field of view move farther away from the boresight target, in a direction that is perpendicular to the JPSS groundtrack, the difference in viewing zenith angles to $P$ and to $Q$ becomes greater, and the measurement becomes less useful for the purpose of intercalibration.

Alignment of the ISS sensor boresight with the line of sight $Q P$ (see Figure 1) is facilitated by attaching the instrument to the ISS with a gimbal that permits rotational motion about two orthogonal axes. Here we use a particular configuration of gimbal axes, the first of which is parallel to the ISS $z$ (or yaw) axis, and the second of which is parallel to the instrument's $x$ (or roll) axis. The angular displacements about the yaw and roll axes are denoted, respectively, by $q_{1}$ and $q_{2}$. The yawroll gimbal sequence is attractive because the value of $q_{1}$ needed to aim the boresight also makes the ISS instrument swath parallel to the swath of the instrument being intercalibrated. Nominally, the ISS instrument scan plane is perpendicular to the ISS ground track when $q_{1}=0^{\circ}$, whereas the scan plane is parallel to the ground track for $q_{1}=90^{\circ}$. The effective swath width is, in the first instance (with $q_{2}=0^{\circ}$ ), $100 \mathrm{~km}$, and, in the second case, $0.5 \mathrm{~km}$. Measurements associated with a narrow effective swath are not useful for intercalibration. In this study the values of $q_{1}$ and $q_{2}$ are calculated under the assumption that the ISS $x, y$, and $z$ axes are parallel to the axes of a local-vertical-local-horizontal reference frame. The ISS Torque Equilibrium Attitude (TEA) differs from this idealization, of course, but each of the three TEA angles is typically expected to be less than $15^{\circ}$ and to vary slowly (Reference 18 ). The values of $q_{1}$ and $q_{2}$ reported here are therefore expected to be a good first approximation, and moderate adjustments to those values will enable the instrument gimbal to compensate for ISS attitude motion.

For the sake of simplicity it is assumed in this paper that no part of the ISS structure obstructs the instrument field of view. Depending on the instrument mounting location, varying levels of partial view obstruction may at times occur (Reference 19). With a particular site specified for the instrument, a corresponding reduction in measurements (and sampling numbers) can be determined.

Simulations of orbital motion during a period of one year have been performed to examine intercalibration opportunities between ISS and two other spacecraft, JPSS and MetOp, which are in near-polar, sun-synchronous orbits that pass through their respective ascending nodes at local times of 13:30 (afternoon) and 21:30 (night). Orbital parameters for each spacecraft are presented in Table 3 .

Table 3. Orbital Parameters of ISS, JPSS, and MetOp

\begin{tabular}{|c|c|c|c|c|c|c|}
\hline \multirow{2}{*}{$\begin{array}{l}\text { Orbital Parameter } \\
\text { altitude }(\mathrm{km})\end{array}$} & \multicolumn{2}{|r|}{ ISS } & \multicolumn{2}{|c|}{$\begin{array}{r}\text { JPSS } \\
(13: 30 \text { local time })\end{array}$} & \multicolumn{2}{|c|}{$\begin{array}{r}\text { MetOp } \\
(21: 30 \text { local time })\end{array}$} \\
\hline & (constant) & 400 & (constant) & 833 & (constant) & 817 \\
\hline inclination, $i$ (deg) & (constant) & 51.6 & (constant) & 98.74 & (constant) & 98.68 \\
\hline right ascension, $\Omega$ (deg) & $(t=0)$ & 0 & $(t=0)$ & 202.5 & $(t=0)$ & 322.5 \\
\hline arg. of latitude, $\nu(\mathrm{deg})$ & $(t=0)$ & 0 & $(t=0)$ & 0 & $(t=0)$ & 0 \\
\hline
\end{tabular}




\section{ORBITAL SIMULATION RESULTS}

In what follows, orbital simulation results are presented in several plots, accompanied by discussion. The plots illustrate the ISS groundtrack during each intercalibration opportunity, a time history of groundtrack latitude at the beginning of each opportunity, the ISS instrument swath during two particular opportunities, time histories of opportunity duration, envelopes of angles characterizing viewing geometry, and envelopes of gimbal angles, along with first and second time-derivatives of the gimbal angles.

Figure 2 shows the ISS groundtrack during each opportunity to take measurements for intercalibrating JPSS sensors. The number of opportunities in one year is determined to be 790 . The groundtracks of course remain within $51.6^{\circ}$ of the equator, but are otherwise well distributed in longitude as well as latitude, which is an indication that the measurements are also well distributed geographically. The length of the groundtrack is proportional to the duration of the opportunity. The number of opportunities over the year to intercalibrate MetOp is found to be 772; ISS groundtracks during these opportunities are displayed in Figure 3.

The latitude of the ISS subsatellite point at the beginning of each opportunity for JPSS intercalibration is plotted over the course of one year in Figure 4, where time $t=0$ corresponds to the instant of autumnal equinox. The sinusoidal behavior has a period of 60 days, which corresponds to the length of the cycle in nodal alignments for the ISS and a sun-synchronous orbit. A similar plot has been constructed for MetOp, but is omitted in the interest of conciseness. As one would expect, the main difference in the two plots consists of a phase shift corresponding to the difference in local times of the nodal crossings of JPSS and MetOp.

Details of the ISS instrument swath during two intercalibration opportunities are provided in Figure 5. Dash-dot curves indicate the ISS ground tracks; a diamond and a square, respectively, mark the beginning and end of each opportunity. The boresight of the ISS instrument is directed along the track marked by solid circles; at every point on this curve the line of sight to ISS is the same as it is to JPSS. The boundaries of the ISS instrument swath are shown with dashed curves. (When the ISS instrument is directed at nadir, the width of the swath is $100 \mathrm{~km}$.)

The duration of each JPSS intercalibration opportunity is shown in Figure 6, depending on whether solar zenith angle $\theta_{0}$ is not or is taken into account. According to Reference 20, measurements of reflected solar radiation are useful for intercalibration only when $\theta_{0} \leq 75^{\circ}$. This constraint is left out of account in the upper plot of Figure 6, whereas the constraint is applied at the ISS instrument boresight target in determining the durations shown in the lower plot. The total yearly durations are 1.41 days and 1.36 days, respectively, without and with the constraint. The 60day nodal alignment cycle is evident in these results. The opportunities having the longest durations, nearly $300 \mathrm{sec}$, occur over near-equatorial latitudes as both spacecraft are ascending or descending together through the equatorial plane. Secondary maxima of approximately $240 \mathrm{sec}$ occur at times when one spacecraft is ascending and the other is descending through the equatorial plane. The minima with values of around $180 \mathrm{sec}$ correspond to opportunities taking place over high latitudes, where the angle between the two orbit planes is greatest.

Durations of opportunities to intercalibrate MetOp sensors are plotted in Figure 7. In the case of MetOp, the total yearly opportunity duration is 1.34 days in the absence of a solar zenith angle constraint, whereas it decreases to 1.23 days when the constraint is applied.

Three viewing angles are important in connection with measuring solar radiation reflected from 


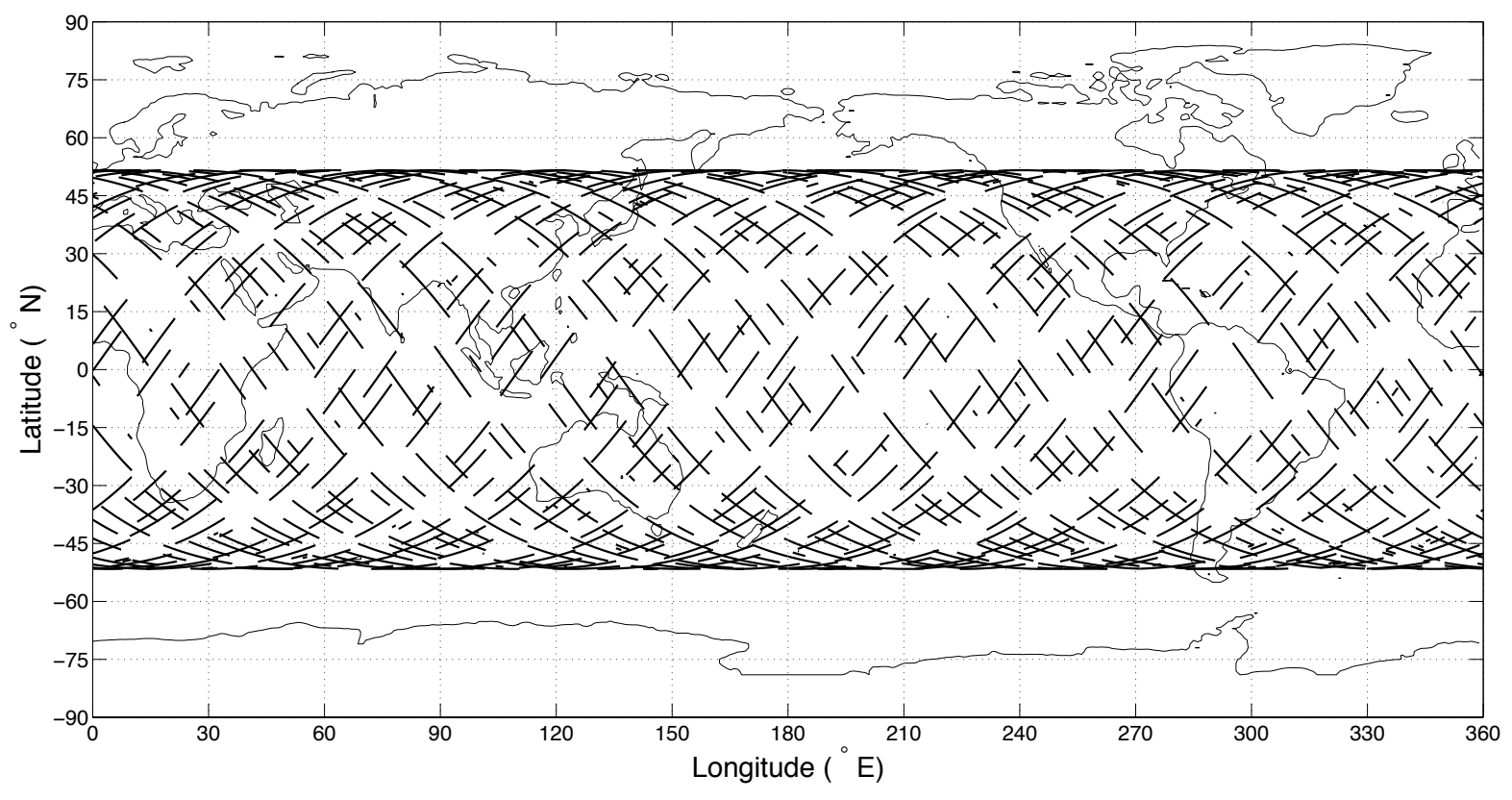

Figure 2. One-Year Distribution of 790 Intercalibration Opportunities, ISS vs. JPSS

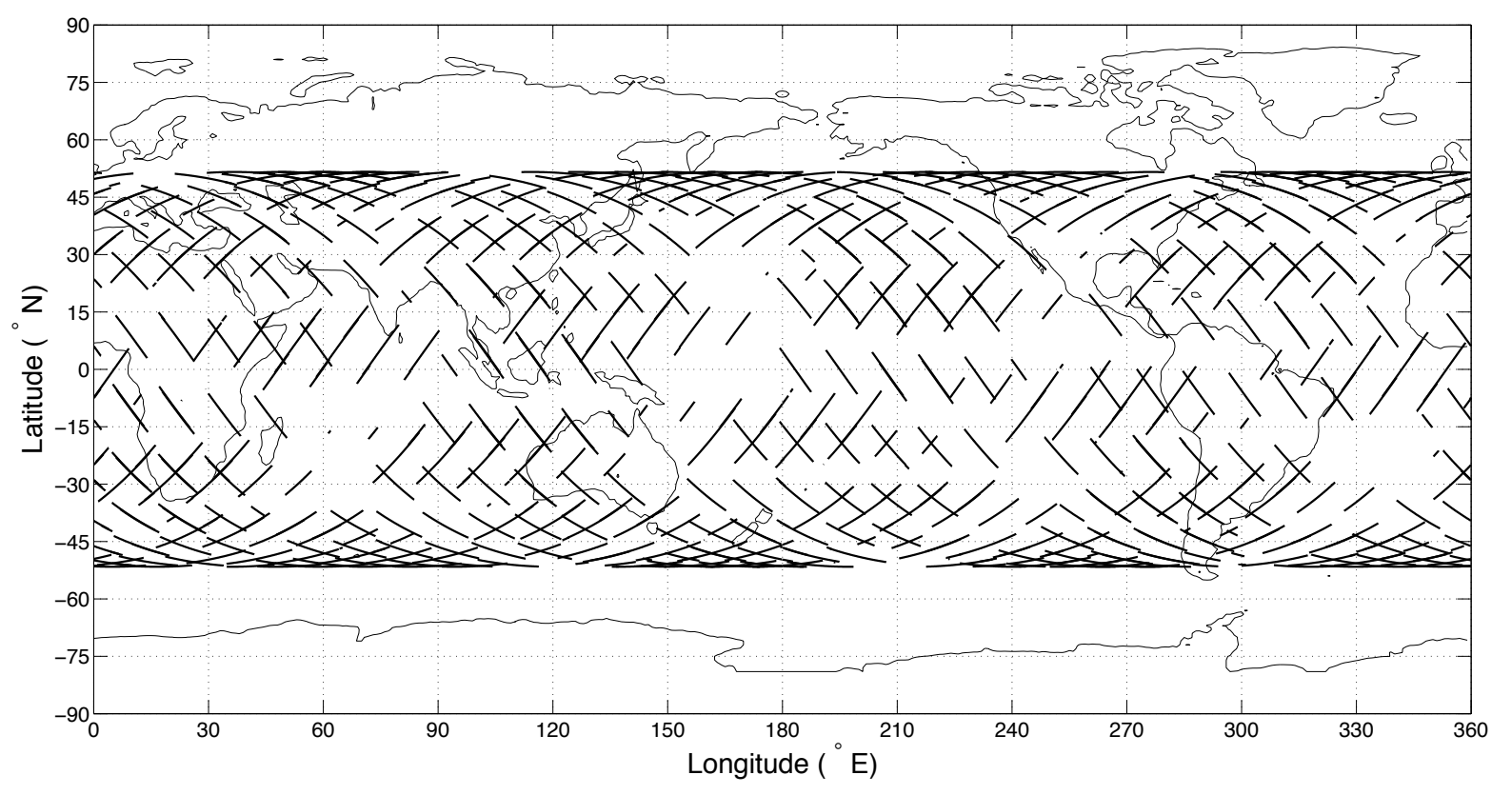

Figure 3. One-Year Distribution of 772 Intercalibration Opportunities, ISS vs. MetOp 


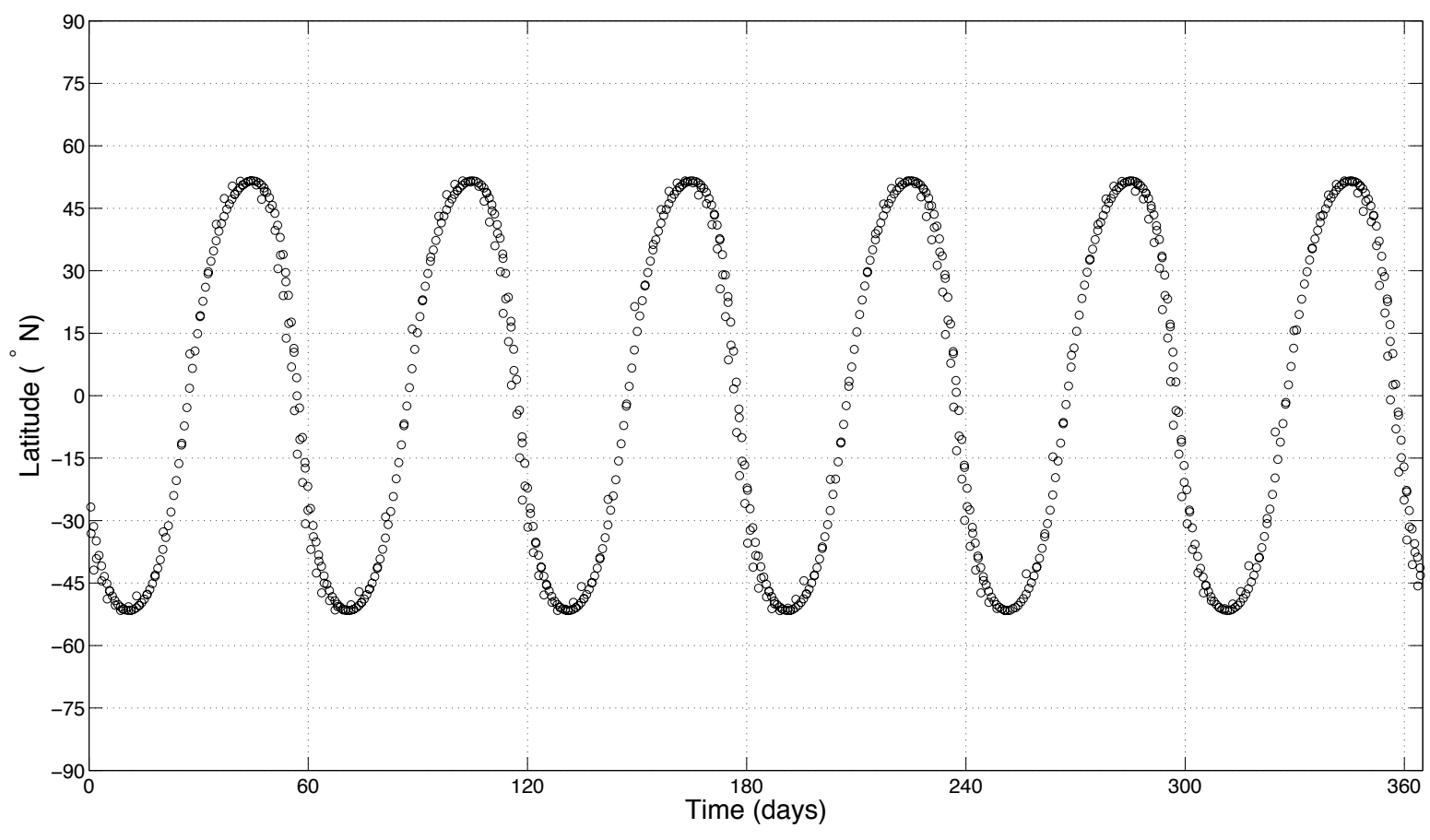

Figure 4. ISS Latitude at the Start of Each Opportunity, JPSS

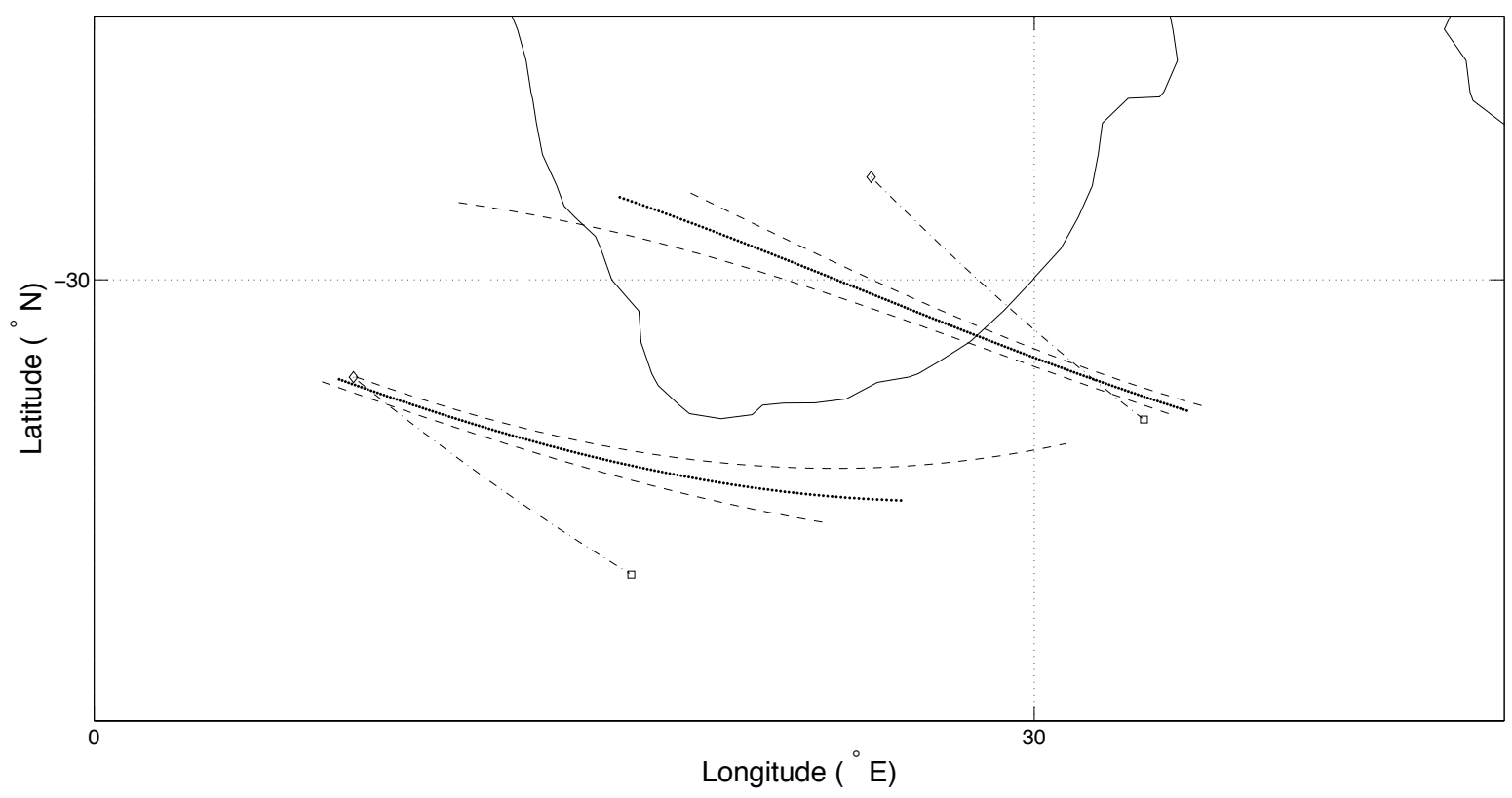

Figure 5. ISS Instrument Swaths for Two Intercalibration Opportunities, JPSS 

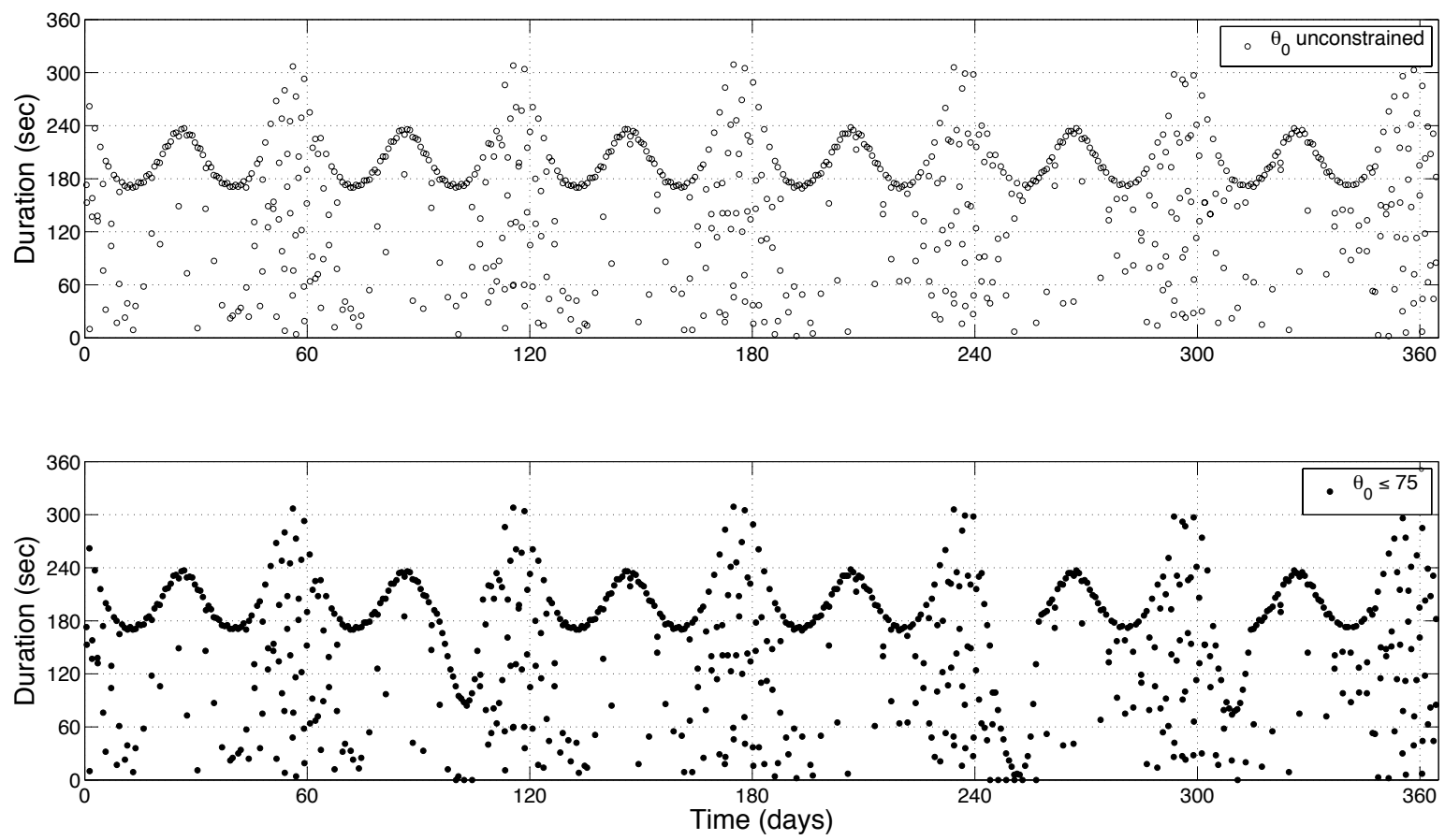

Figure 6. Durations of Intercalibration Opportunities, ISS vs. JPSS
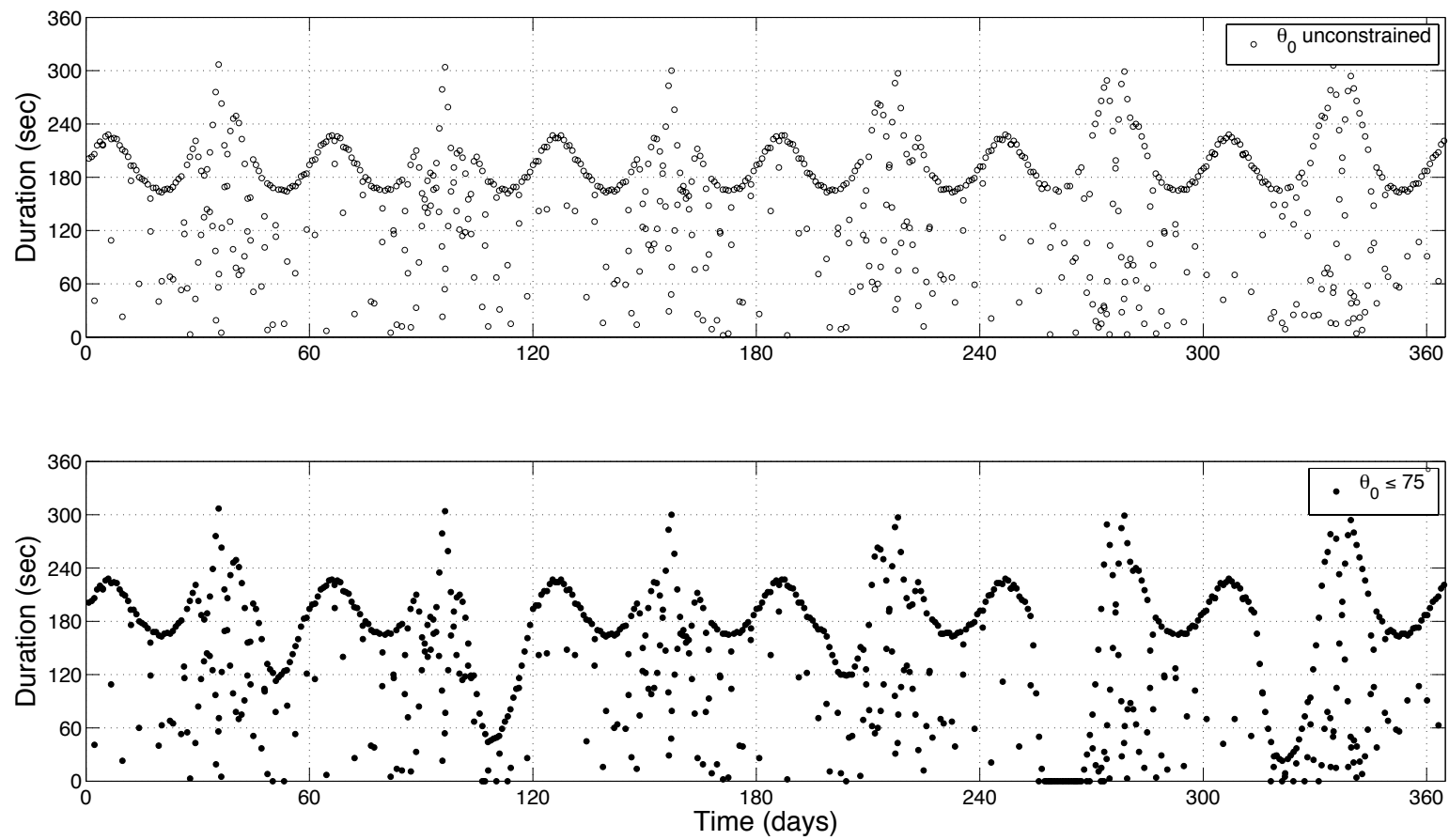

Figure 7. Durations of Intercalibration Opportunities, ISS vs. MetOp 

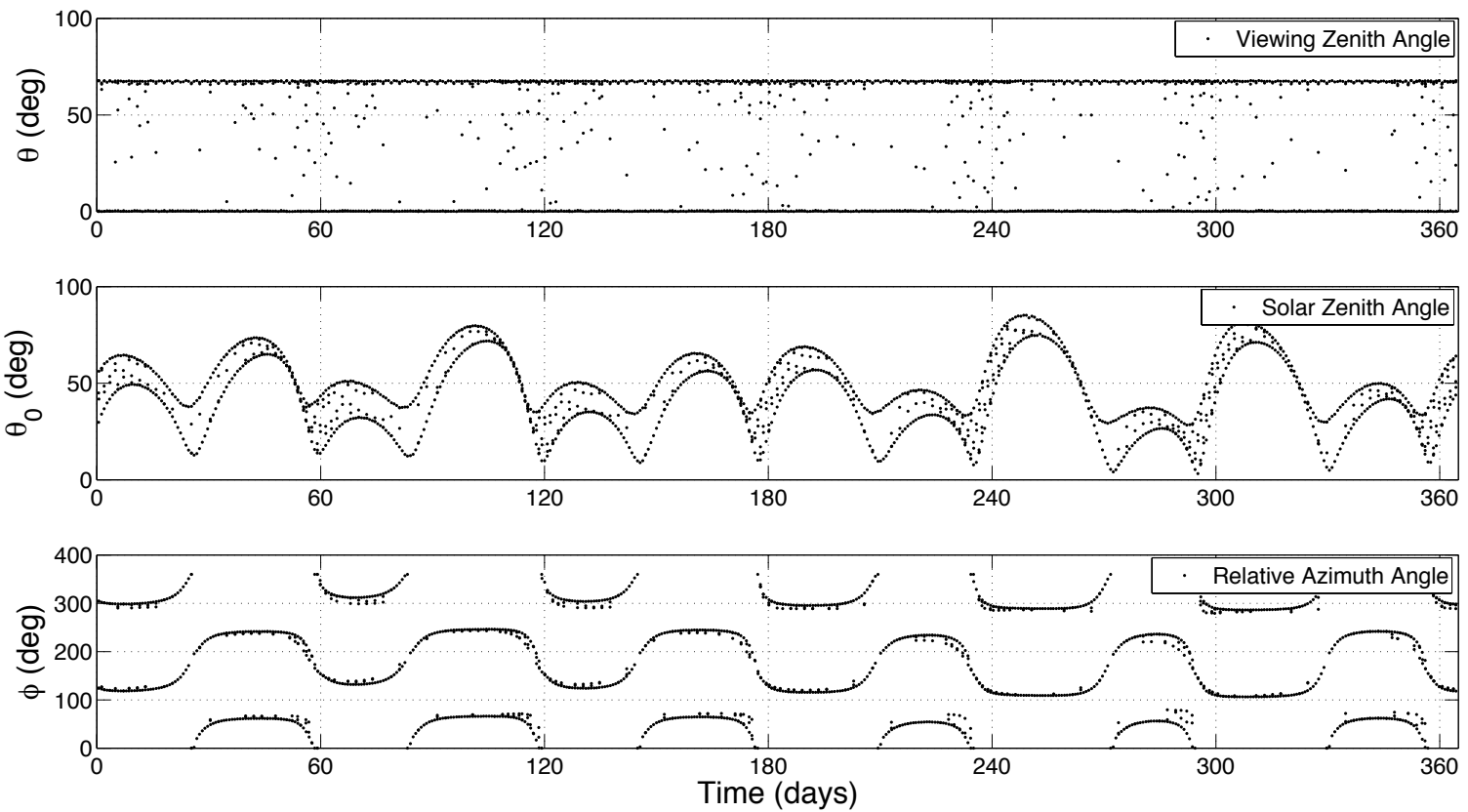

Figure 8. Viewing Angles at Boresight Target, Min and Max Values in Each Opportunity, ISS vs. JPSS

a point $T$ on Earth's surface. Two of the angles have already been introduced: the viewing zenith angle, $\theta$, and the solar zenith angle, $\theta_{0}$. The third angle, referred to as the relative azimuth angle, $\phi$, is measured between two planes that intersect on the local zenith line. One of the planes contains the line of sight from $T$ to a spacecraft, and the other plane contains the line of sight from $T$ to the Sun. Values of $\phi$ range from 0 to $360^{\circ}$.

Maximum and minimum values of all three viewing angles at the ISS instrument boresight target are plotted in Figure 8 for each JPSS intercalibration opportunity over a year. The upper bound on viewing zenith angle is about $68^{\circ}$. Solar zenith angle has a global maximum of approximately $85^{\circ}$, and exceeds $75^{\circ}$ on three occasions for several days each. It is during these three intervals that the useful durations drop noticeably in the lower plot of Figure 6. For the first 30 days or so, the relative azimuth angle ranges roughly between $120^{\circ}$ and $300^{\circ}$. Over the next 30-day period the bounds are approximately $60^{\circ}$ and $240^{\circ}$. This pattern repeats itself thereafter. A $180^{\circ}$ difference between maximum and minimum is indicative of a discontinuity in relative azimuth angle as ISS passes under the ridge of the tent (see Figure 1) during a typical opportunity. Plots of viewing angle extrema for MetOp intercalibration opportunities are similar to those in Figure 8, and are omitted in the interest of brevity.

During each JPSS intercalibration opportunity, the ISS instrument gimbal must move such that the boresight remains parallel to the line of sight from JPSS to ISS. Behavior of the yaw gimbal is illustrated in Figure 9 where, for each opportunity, the maximum and minimum gimbal angle $q_{1}$, angular speed $\dot{q}_{1}$, and angular acceleration $\ddot{q}_{1}$ are shown in the upper, middle, and lower plots, respectively. The 60-day nodal alignment cycle is once again apparent. The yaw gimbal is not required to move much during each opportunity, as can be seen by inspecting the plots for $q_{1}$ and 

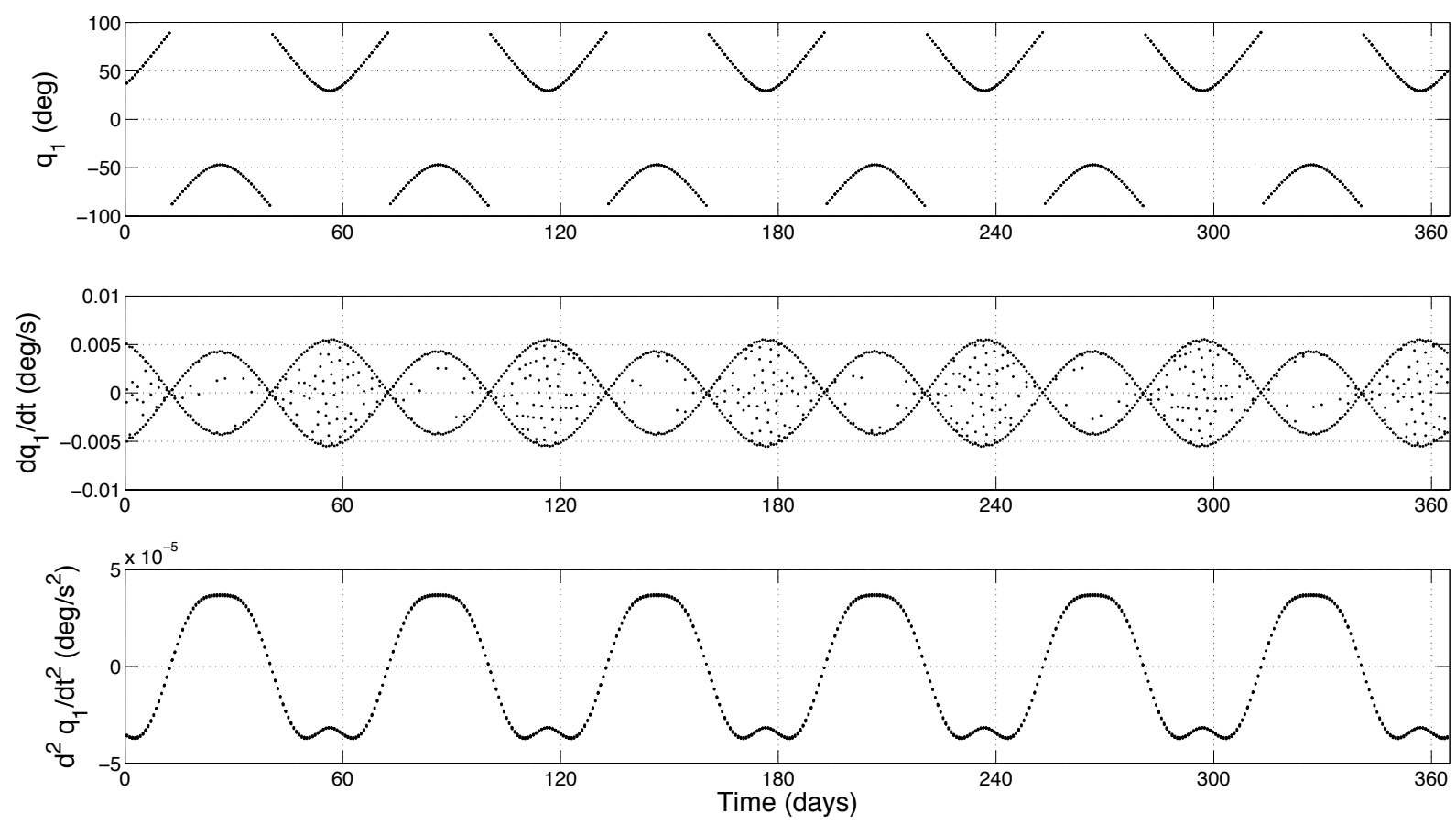

Figure 9. Yaw Gimbal Motion, Min and Max Values in Each Opportunity, ISS vs. JPSS
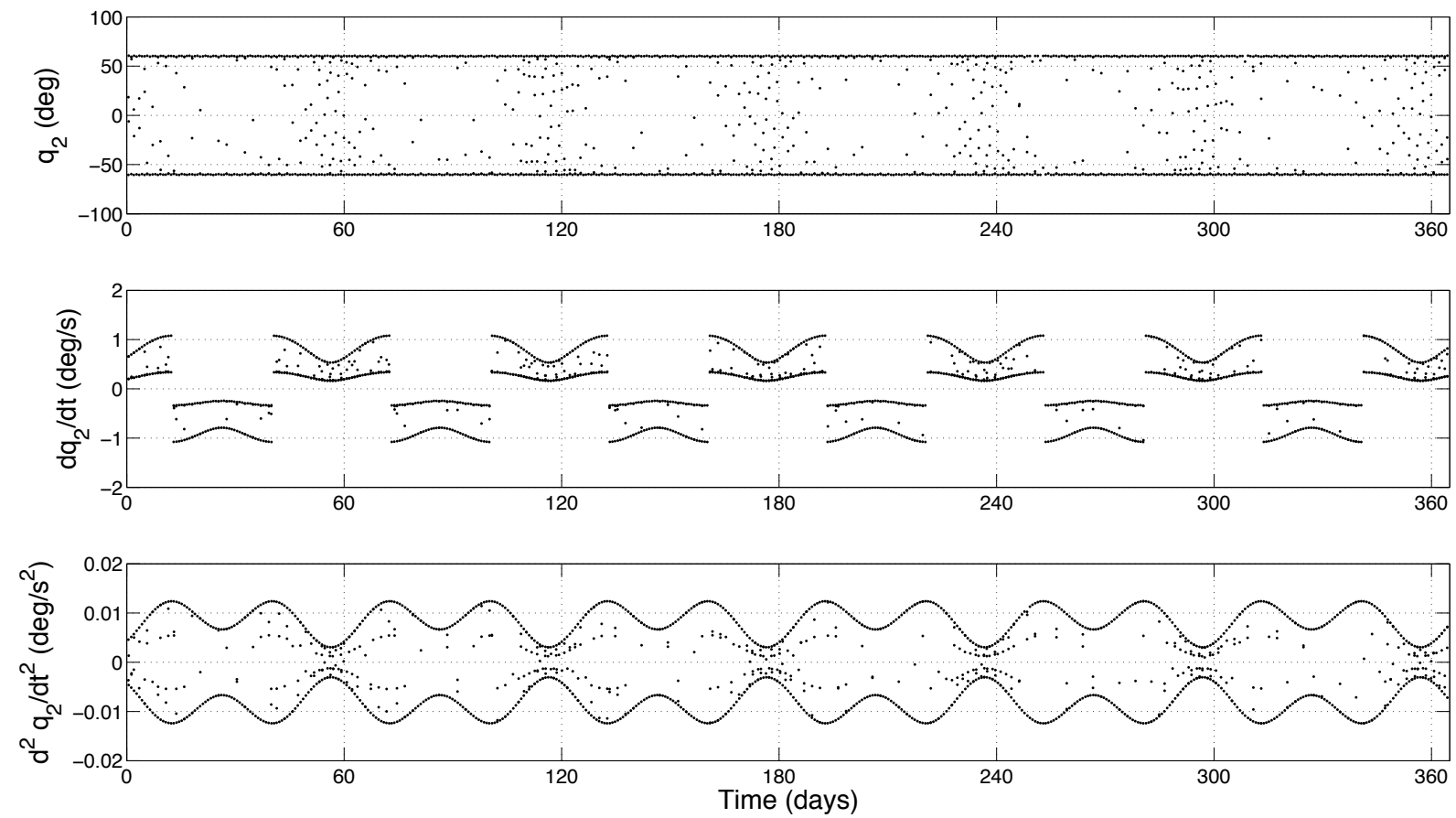

Figure 10. Roll Gimbal Motion, Min and Max Values in Each Opportunity, ISS vs. JPSS 

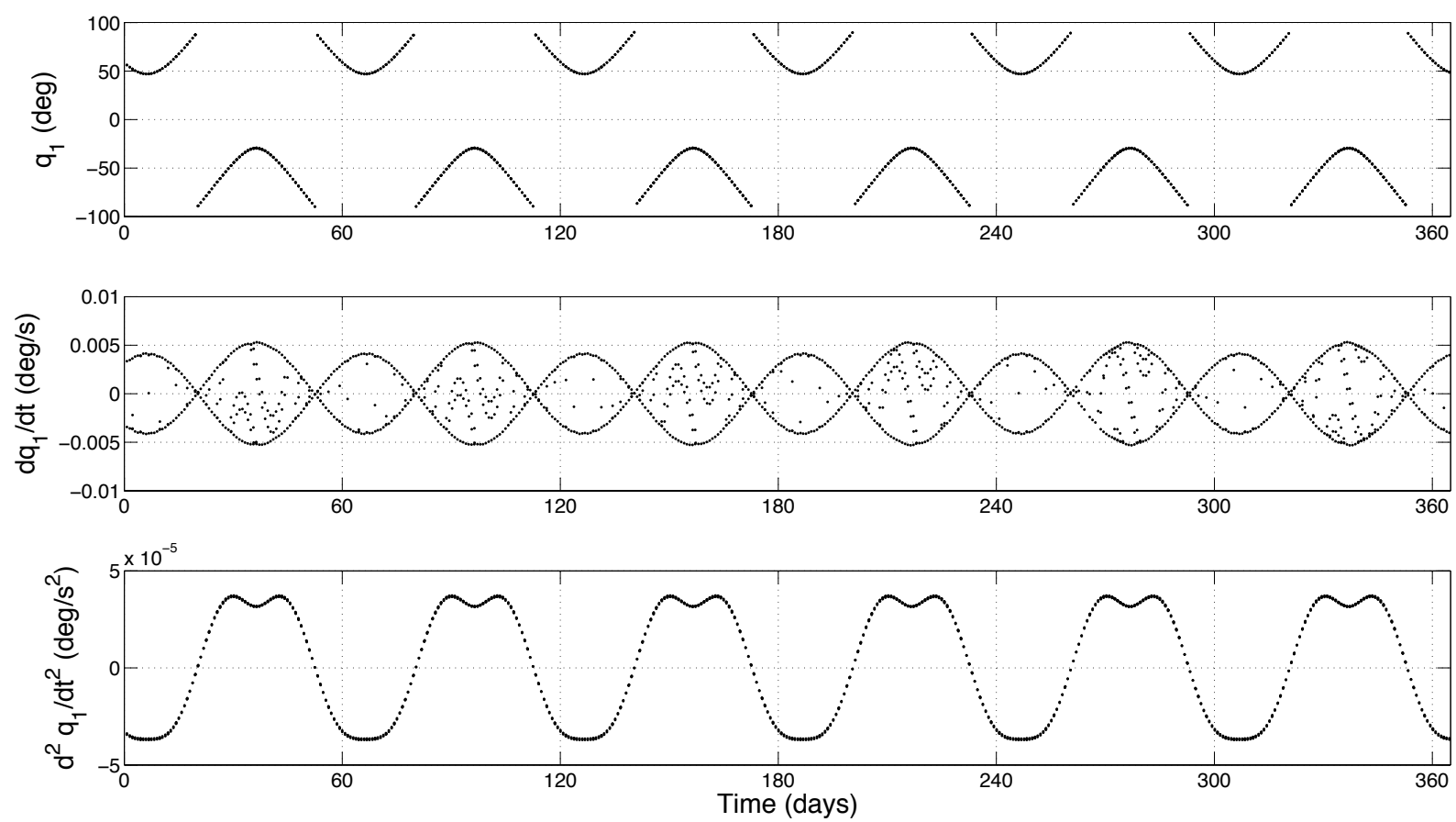

Figure 11. Yaw Gimbal Motion, Min and Max Values in Each Opportunity, ISS vs. MetOp
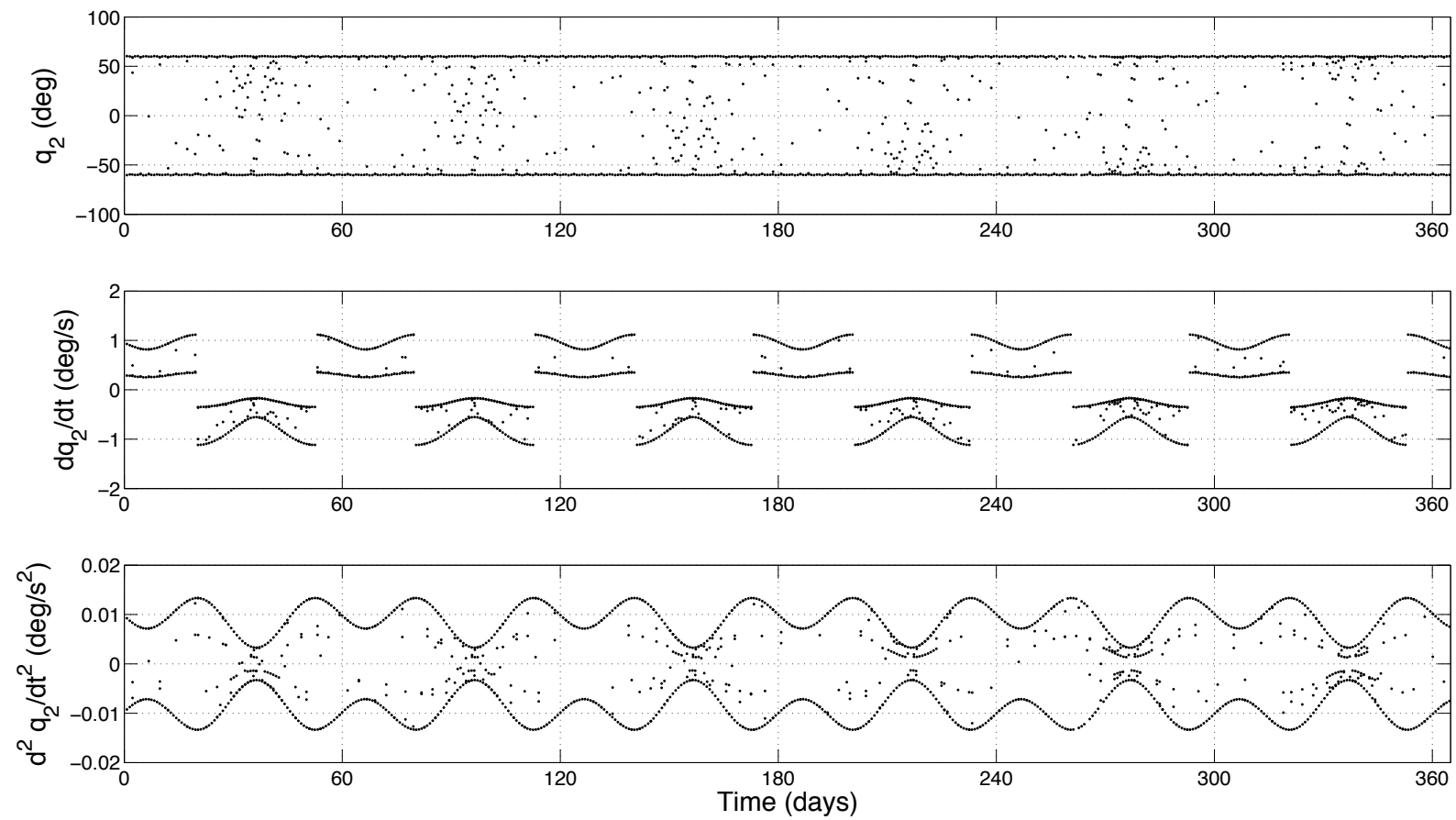

Figure 12. Roll Gimbal Motion, Min and Max Values in Each Opportunity, ISS vs. MetOp 
$\dot{q}_{1}$. The gimbal angular speed need not exceed $0.006 \%$ in absolute value. On the other hand, Figure 10 shows the roll gimbal will need to be more active than the yaw gimbal. Excursions in $q_{2}$ during each opportunity will typically be about $110^{\circ}$, which is twice the value of the scan angle of the instruments on JPSS. The roll gimbal will be required to produce an angular speed $\dot{q}_{2}$ of around $1.1 \% \mathrm{~s}$ in absolute value. The plot of $\ddot{q}_{2}$ indicates a maximum of about $0.013 \% \mathrm{~s}^{2}$ is needed. Torque motors for the yaw and roll gimbals can be sized according to the global maximum values of $\ddot{q}_{1}$ and $\ddot{q}_{2}$ obtained from Figures 9 and 10, respectively.

Behavior of the yaw and roll gimbals required for MetOp intercalibration opportunities is shown in Figures 11 and 12, respectively. The chief differences in comparison to Figures 9 and 10 consist of phase shift, as well as reflection about the horizontal axis in some cases. These differences are attributable to the difference in local times of the nodal crossings for the JPSS and MetOp orbits.

As mentioned previously, some adjustments to Figures $9-12$ are necessary to account for ISS TEA motion.

The envelopes of $\dot{q}_{i}$ and $\ddot{q}_{i}(i=1,2)$ presented in Figures $9-12$ lie inside those associated with a nominal CLARREO orbit (altitude $609 \mathrm{~km}$, inclination $90^{\circ}$ ). The difference in orbital altitudes of CLARREO and JPSS was approximately $200 \mathrm{~km}$, whereas the altitudes of ISS and JPSS differ by about $400 \mathrm{~km}$; the line of sight between spacecraft changes direction more gradually in the latter pairing.

\section{REFERENCE INTERCALIBRATION SAMPLING}

Data matching noise is reduced by forming intercalibration samples from measurements averaged over some amount of Earth surface area. The sensitivity of matching noise to differences in time, horizontal space, and viewing angles is established in Reference 17 by using analogs of planned CLARREO intercalibration procedures. It was found that instantaneous data noise is limited to $1 \%$ or less by keeping the differences in viewing angles $\left(\theta, \theta_{0}\right.$, and $\left.\phi\right)$ within $1.5^{\circ}$, and limiting the difference in time of measurements to 5 minutes or less. These matching constraints were determined using three months of data from AVHRR NOAA 17 and NOAA 18 orbit crossings.

Here, we construct intercalibration samples for the VIIRS and AVHRR instruments such that spatial matching noise is limited to $1 \%$. This is accomplished by defining a sample in angular space as $1.5^{\circ}$ of elevation angle, which corresponds from the ISS orbit to a nadir equivalent area of 10 $\mathrm{km} \times 10 \mathrm{~km}$. Two samples having independent spatial noise are related to each other by a shift of $0.15^{\circ}$ in elevation angle, which results in a 1-km shift in each of two spatial directions, along and perpendicular to the ground track. The CLARREO spatial resolution of $0.5 \mathrm{~km} \times 0.5 \mathrm{~km}$ ensures the two samples have only two boundary pixels in common. We estimate the number of samples by shifting them in this way. (It should be noted that this approach to forming samples does not

allow intercalibration on a detector-by-detector basis. Relative calibration of VIIRS detectors to each other requires use of VIIRS data alone; the same is true of AVHRR detectors.)

The number of CLARREO samples that can be used to intercalibrate CERES is estimated by taking into account a CERES nadir footprint size of $25 \mathrm{~km}$ from the JPSS orbit, and a data acquisition rate of 330 footprints in each $180^{\circ}$ scan performed over 3.3 seconds.

Using the orbital simulation results presented in the previous section, we estimate the projected number of intercalibration samples for VIIRS and CERES instruments on the JPSS, and the AVHRR instrument on the MetOp satellite. All estimates are made with the following constraints: (a) $\theta_{0}<$ $75^{\circ}$ (to ensure high Signal-to-Noise Ratio); (b) CLARREO effective swath width is greater than 10 
$\mathrm{km}$ for VIIRS and AVHRR, and greater than $25 \mathrm{~km}$ for CERES; (c) $\theta$ is matched within $1.5^{\circ}, \theta_{0}$ and $\phi$ are matched within $1^{\circ}$; and (d) intercalibration event duration is greater than $10 \mathrm{sec}$. The sampling estimates are presented in Figure 13, reported by month in the upper plot, and by season in the lower plot. Comparison of these estimates with Table 2 shows that the numbers of samples are more than sufficient to intercalibrate well-behaved sensors in JPSS and MetOp orbits to the accuracy required for measuring long-term climate change. Over a climate autocorrelation time period of 18 months, the intercalibration error contribution can be contained within $0.3 \%(k=2)$.
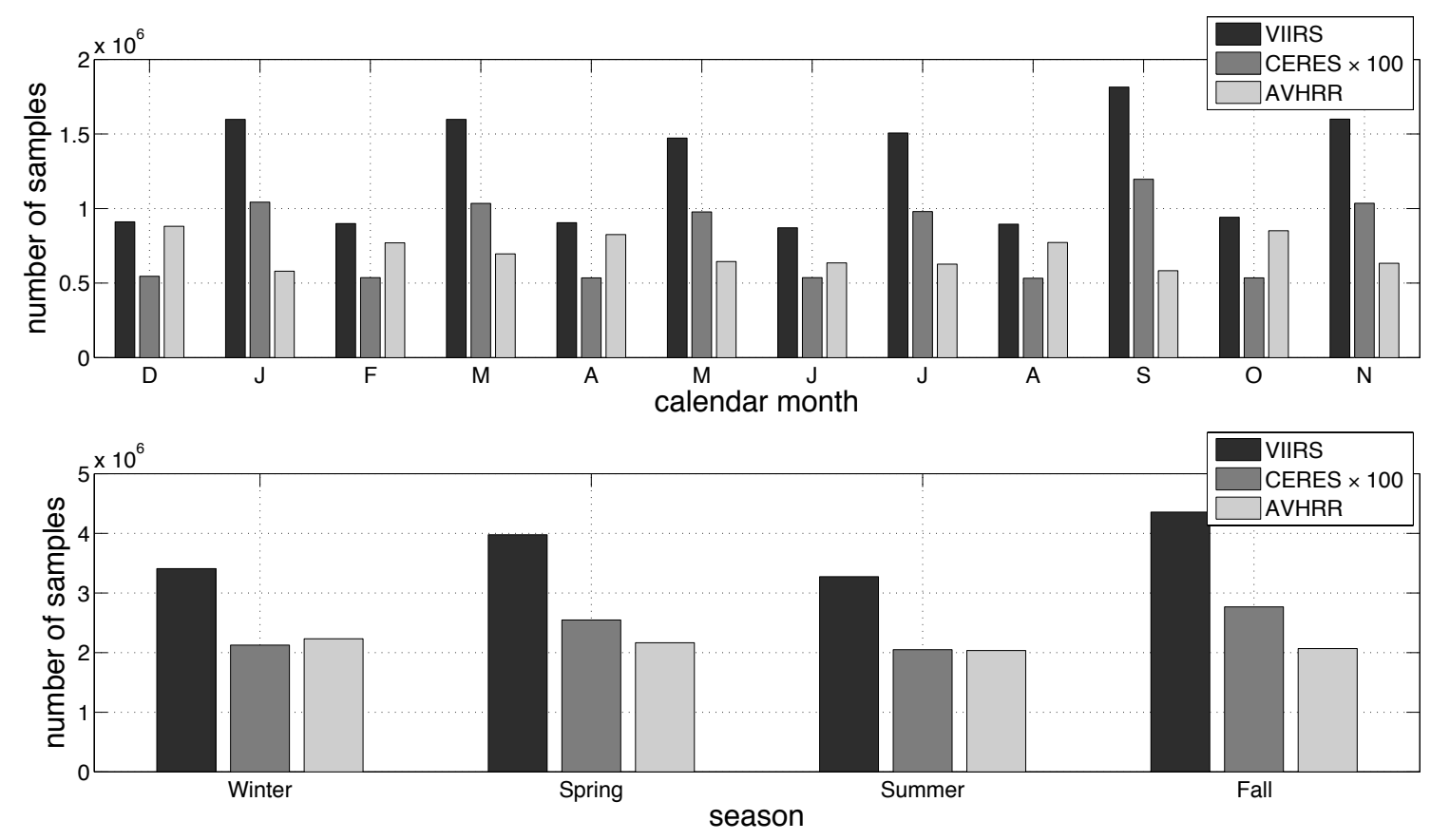

Figure 13. Monthly and Seasonal Sampling Estimates, ISS vs: JPSS-VIIRS, JPSSCERES, and MetOp-AVHRR

\section{CONCLUSIONS}

The International Space Station is ideally suited to serve as a platform from which to obtain reflected solar radiance measurements that can be used to intercalibrate instruments in sun-synchronous low Earth orbits. The ISS orbit provides coverage of a large part of the globe, which extends from $51.6^{\circ}$ South to $51.6^{\circ}$ North latitude. All scene types necessary for intercalibration, including clouds, snow, clear-sky ocean, desert, and vegetation, can be found within the area of coverage. Results of orbital simulations show that the difference in ISS and sun-synchronous orbit plane precession leads to temporal uniformity in opportunities for intercalibration. Angular speed and angular acceleration required from a two-degree-of-freedom instrument gimbal for matching line of sight on ISS compares favorably to what is required for a dedicated CLARREO mission.

The numbers of samples that can be obtained from ISS are more than sufficient to intercalibrate well-behaved sensors in sun-synchronous low Earth orbits to the accuracy required for measuring long-term climate change. The intercalibration error contribution can be kept within $0.3 \%(k=$ 2 ) over a climate autocorrelation time period of 18 months. Using a CLARREO reflected solar 
sensor as a high-accuracy orbiting standard for comprehensive reference intercalibration of other instruments would improve their accuracy substantially, and thereby add significant value to the existing Earth Observing System.

\section{REFERENCES}

[1] Wielicki, B. A., et al., "Climate Absolute Radiance and Refractivity Observatory (CLARREO): Achieving Climate Change Absolute Accuracy in Orbit," Bulletin of the American Meteorological Society, in preparation.

[2] Earth Science and Applications from Space: National Imperatives for the Next Decade and Beyond, National Research Council (U.S.), Committee on Earth Science and Applications from Space, National Academies Press, 2007, pp. 92-95.

[3] Fox, N., Kaiser-Weiss, A., Schmutz, W., Thome, K., Young, D., Wielicki, B., Winkler, R., and Woolliams, E., "Accurate Radiometry from Space: An Essential Tool for Climate Studies, Philosophical Transactions of The Royal Society A: Mathematical, Physical, and Engineering Sciences, Vol. 369, No. 1953, 2011, pp. 4028-4063.

[4] Goldberg, M., et al., "The Global Space-Based Inter-Calibration System," Bulletin of the American Meteorological Society, Vol. 92, 2011, pp. 467-475.

[5] Research Announcement for International Space Station Experiments relevant to study of Global Climate Change, European Space Agency, Directorate of Human Spaceflight and Operations, Directorate of Earth Observation Programmes, Noordwijk, The Netherlands, July 7, 2011.

[6] Announcement of Opportunity, Earth System Science Pathfinder (ESSP) Program, Earth Venture - 2, NNH11ZDA012O, National Aeronautics and Space Administration, Science Mission Directorate, June 17, 2011.

[7] Roithmayr, C. M., Lukashin, C., Speth, P. W., Thome, K. J., Wielicki, B. A., and Young, D. F., "Measurements of Reflected Solar Radiation by CLARREO for Reference Intercalibration," IEEE Transactions on Geoscience and Remote Sensing, in preparation.

[8] Evaluation of Measurement Data - Guide to the Expression of Uncertainty in Measurement, Joint Committee for Guides in Metrology, Bureau International des Poids et Mesures, 2008 (Corrected version 2010).

[9] Leroy, S. S., Anderson, J. G., and Ohring, G., "Climate Signal Detection Times and Constraints on Climate Benchmark Accuracy Requirements," Journal of Climate, Vol. 21, No. 4, 2008, pp. 841-846.

[10] Clark, L. G., and DiBattista, J. D., "Space Qualification Of Optical Instruments Using The NASA Long Duration Exposure Facility," Optical Engineering, Vol. 17, No. 5, 1978, pp. 547-552.

[11] Wielicki, B. A., Barkstrom, B. R., Harrison, E. F., Lee, R. B., Smith, G. L., and Cooper, J. E., "Clouds and the Earth's Radiant Energy System (CERES): An Earth Observing System Experiment," Bulletin of the American Meteorological Society, Vol. 77, No. 5, 1996, pp. 853-868.

[12] Sun, J.-Q., and Xiong, X., "MODIS Polarization-Sensitivity Analysis," IEEE Transactions on Geoscience and Remote Sensing, Vol. 45, No. 9, 2007, pp. 2875-2885.

[13] Nadal, F., and Bréon, F.-M., "Parameterization of Surface Polarized Reflectance Derived from POLDER Spaceborne Measurements," IEEE Transactions on Geoscience and Remote Sensing, Vol. 37, No. 3, 1999, pp. 1709-1718.

[14] Xiong, X., Che, N., Pan, C., Xie, X., Sun, J., Barnes, W. L., and Guenther, B., "Results and Lessons from MODIS Reflective Solar Bands Calibration: Pre-launch to On-orbit," Proceedings of SPIE Earth Observing Systems XI, Vol. 6296, 629607, 2006.

[15] Xiong, X., Che, N., and Barnes, W. L., "Terra MODIS On-Orbit Spectral Characterization and Performance," IEEE Transactions on Geoscience and Remote Sensing, Vol. 44, No. 8, 2006, pp. 2198-2206.

[16] Roithmayr, C. M., and Speth, P. W., "Analysis of Opportunities for Intercalibration between Two Spacecraft," Chap. 13, Advances in Engineering Research, Vol. 1, edited by V. M. Petrova, Nova Science Publishers, Hauppauge, NY, 2012, pp. 409-436.

[17] Wielicki, B. A., Doelling, D. R., Young, D. F., Loeb, N. G., Garber, D. P., and MacDonnell, D. G., "Climate Quality Broadband and Narrowband Solar Reflected Radiance Calibration Between Sensors in Orbit," International Geoscience and Remote Sensing Symposium (IGARSS), Vol. 1, 2008, pp. I-257I-260.

[18] Gomez, S., "GNC Performance Requirements and Predicted Performance Estimates," EG-DIV-10-040, NASA Johnson Space Center, Nov. 19, 2010.

[19] Overview of Attached Payload Accommodations and Environments on the International Space Station, TP-2007-214768, NASA Johnson Space Center, September, 2007. 
[20] Minnis, P., Doelling, D. R., Nguyen, L., Miller, W. F., and Chakrapani, V., "Assessment of the Visible Channel Calibrations of the VIRS on TRMM and MODIS on Aqua and Terra,"Journal of Atmospheric and Oceanic Technology, Vol. 25, No. 3, 2008, pp. 385-400. 